Zero pot ent i al energy criteri on appl i ed to Hart ree Fock wave f uncti on $s$

\begin{tabular}{|c|c|}
\hline 著者 & KOGA Toshi kat su \\
\hline $\begin{array}{l}\text { jour nal or } \\
\text { publ i cat } i \text { on } \mathrm{titl} e\end{array}$ & The j our nal of chemi cal physi cs \\
\hline vol une & 89 \\
\hline nunber & 7 \\
\hline page $r$ ange & 4209- 4211 \\
\hline year & $1988-10-01$ \\
\hline URL & ht t p: //hdl . handl e. net /10258/932 \\
\hline
\end{tabular}




\title{
Zero potential energy criterion applied to Hartree-Fock wave functions
}

\author{
Toshikatsu Koga \\ Department of Applied Chemistry and Department of Applied Science for Energy, Muroran Institute of \\ Technology, Muroran, Hokkaido 050, Japan
}

(Received 1 March 1988; accepted 24 June 1988)

\begin{abstract}
The zero potential energy criterion, which is a necessary condition for the exact wave function, is applied to Hartree-Fock wave functions for a closed-shell system with doubly occupied spatial orbitals. With the help of the known long-range asymptotic behavior of Hartree-Fock orbitals, we first derive a single-electron zero potential energy criterion to be satisfied by Hartree-Fock orbitals. The Hartree-Fock wave function is then shown to never satisfy the zero potential energy criterion, which implies that the Hartree-Fock approximation cannot describe the correct long-range asymptotic behavior of many-electron wave functions. Some numerical illustrations are given.
\end{abstract}

\section{INTRODUCTION}

Previously, the zero potential energy $\left(E_{\mathrm{up}}\right)$ criterion has been proposed ${ }^{\prime}$ and applied ${ }^{1-3}$ as a sensitive measure for assessing the accuracy of approximate wave functions. $E_{\mathrm{zp}}$ is defined as the local energy ${ }^{4-6}$ evaluated at those points in position space at which the potential energy operator $V\left(\left\{\mathbf{r}_{i}\right\}\right)$ vanishes, i.e.,

$$
E_{\text {ap }}=\lim _{\boldsymbol{V} \sim 0}\left\{\left[T\left(\left\{\mathbf{r}_{i}\right\}\right) \Psi\left(\left\{\mathbf{r}_{i}\right\}\right)\right] / \Psi\left(\left\{\mathbf{r}_{i}\right\}\right)\right\},
$$

where $\Psi\left(\left\{\mathbf{r}_{i}\right\}\right)$ is the total electronic wave function, $\left\{\mathbf{r}_{i}\right\}$ the position vectors of electrons, and $T\left(\left\{\mathbf{r}_{i}\right\}\right)$ the electronic kinetic energy operator $T\left(\left\{\mathbf{r}_{i}\right\}\right)=\Sigma_{i} t\left(\mathbf{r}_{i}\right)$, where $t\left(\mathbf{r}_{i}\right)$ $=-(1 / 2) \Delta_{i}$. The zero potential energy has an important property ${ }^{\prime}$ that the relation

$$
E_{\text {zp }}=E_{\text {av }}=E
$$

is a necessary (but not sufficient) condition for a wave function to be the true wave function, where $E$ is the true energy and $E_{\text {av }}$ the average energy. It is known ${ }^{1}$ that $E_{\text {ap }}$ is more sensitive to the wave function error than $E_{\mathrm{av}}$, since $E_{\mathrm{zp}}$ and $E_{\text {av }}$ are, respectively, linear and quadratic in the error.

The zero potential energy formula, Eq. (1), is a special form of the local energy formula with vanishing potential energy contribution, and it may be regarded as a partner to the zero momentum energy criterion ${ }^{7-10}$ which considers the momentum-space local energy with vanishing kinetic energy contribution. For atoms and molecules of our interest, the limiting procedure $V \rightarrow 0$ involved in Eq. (1) is equivalent to considering a point infinitely apart from the nuclei where Coulombic interactions disappear. Therefore, $E_{\text {zp }}$ measures the quality of wave functions at their long-range tails.

In the present paper, we study the zero potential energy of the Hartree-Fock wave function for a closed-shell system from the viewpoints of the one-electron orbital and the total electronic wave function. In Sec. II, the zero potential consideration is applied to the Fock equation and the orbitalwise zero potential energy criterion is derived based on the known long-range asymptotic behavior ${ }^{11,12}$ (see also Refs. 13-19 and the references therein ). In Sec. III, the total zero potential energy is derived for the Hartree-Fock wave function described as a single Slater determinant. The result shows that the Hartree-Fock approximation cannot correctly de- scribe the long-range behavior of the many-electron wave function. Some numerical examples are presented in Sec. IV. Atomic units are used throughout this paper.

\section{ORBITALWISE ZERO POTENTIAL ENERGY}

Let us consider the Hartree-Fock approximation for a closed-shell $2 N$ electron atomic or molecular system with $N$ doubly occupied spatial orbitals $\left\{\psi_{i}\right\}$. For a large $r$, the leading asymptotic behavior of the Hartree-Fock orbital $\psi_{i}$ is known ${ }^{11,12}$ to be

$$
\psi_{i}(\mathbf{r})=\left[a_{i} r^{b_{i}}+O\left(r^{b_{i}-1}\right)\right] \exp \left(-\zeta_{i} r\right), \quad r=|\mathbf{r}| .
$$

The exponent $\zeta_{i}$ is $\left(-2 \epsilon_{i}\right)^{1 / 2}$ for atoms in which only s-type orbitals appear (referred to as $s$-type atoms hereafter), while otherwise it is $\left(-2 \epsilon_{h}\right)^{1 / 2}$ independent of $i . \epsilon_{i}$ is the orbital energy associated with $\psi_{i}$, and $h$ refers to the highest occupied orbital which is presumed to be nondegenerate. The expression for $b_{i}$ can be found in Ref. 12. Now from Eq. (3), the zero potential energy criterion for the orbital $\psi_{i}$ is obtained as

$$
\begin{aligned}
\epsilon_{\mathrm{zp}, i} & =\lim _{r \rightarrow \infty}\left\{\left[t(\mathbf{r}) \psi_{i}(\mathbf{r})\right] / \psi_{i}(\mathbf{r})\right\}=-(1 / 2) \zeta_{i}^{2} \\
& = \begin{cases}\epsilon_{i} & \text { for } s \text {-type atoms } \\
\epsilon_{h} & \text { otherwise }\end{cases}
\end{aligned}
$$

For $s$-type atoms, the correct Hartree-Fock orbital $\psi_{i}$ must have the zero potential energy equivalent to its orbital energy. The orbitalwise criterion in this special case is identical to that for the Hartree orbital. For other cases, however, the zero potential energy should be equal to the orbital energy of the highest occupied orbital, independent of $i$ ! This curious condition for the Hartree-Fock orbital originates from the nonvanishing contribution of exchange terms at $r \rightarrow \infty$. Indeed, we have for systems other than $s$-type atoms that

$$
\kappa_{i}=\lim _{r \rightarrow \infty}\left\{\left[\sum_{j(\neq i)} K_{j}(\mathbf{r}) \psi_{i}(\mathbf{r})\right] / \psi_{i}(\mathbf{r})\right\}=\epsilon_{h}-\epsilon_{i},
$$

where $K_{j}$ is the exchange operator. At a point infinitely apart 
from the nuclei, the exchange term $\kappa_{i}$ locally has a constant contribution which is directly related to the orbital energy. Equation (4) is the consistency condition for the asymptotic behavior of the Hartree-Fock orbital and the associated orbital energy. Applications of this criterion will be presented in Sec. IV for a few atoms.

\section{HARTREE-FOCK ZERO POTENTIAL ENERGY}

The Hartree-Fock wave function is written as

$$
\begin{aligned}
\Psi\left(\left\{\mathbf{r}_{i}\right\}\right)= & {[(2 N) !]^{-1 / 2} \sum_{P}(-1)^{P} P } \\
& \times\left[\psi_{1}(1) \bar{\psi}_{1}(2) \cdots \psi_{N}(2 N-1) \bar{\psi}_{N}(2 N)\right],
\end{aligned}
$$

where the symbols have the ordinary meaning. The zero potential energy for the wave function (6) is then

$$
E_{z p}=\sum_{i}\left\{\lim _{r_{i} \rightarrow \infty}\left[t\left(\mathbf{r}_{i}\right) \Psi\left(\left\{\mathbf{r}_{i}\right\}\right)\right] / \Psi\left(\left\{\mathbf{r}_{i}\right\}\right)\right\} .
$$

Using the asymptotic form of a spatial function [Eq. (3)], we find for a large $r$ that

$$
t\left(\mathbf{r}_{i}\right) \Psi\left(\left\{\mathbf{r}_{i}\right\}\right)=-(1 / 2) \zeta_{i}^{2} \Psi\left(\left\{\mathbf{r}_{i}\right\}\right) \quad\left(\text { large } r_{i}\right)
$$

and therefore Eq. (7) is reduced to

$$
E_{\mathrm{zp}}= \begin{cases}2 \sum_{i} \epsilon_{i} & \text { for } s \text {-type atoms } \\ 2 N \epsilon_{h} & \text { otherwise }\end{cases}
$$

Equation (9) shows that the Hartree-Fock zero potential energy is the sum of orbital energies of the occupied orbital or the orbital energy of the highest occupied orbital times the number of electrons. It constitutes the consistency condition for the Hartree-Fock wave function and the total electronic energy, deduced from the long-range asymptotic consideration. Evidently, $E_{\text {zp }}$ does not agree with $E_{\mathrm{av}}$ which is given by

$$
E_{\mathrm{av}}=2 \sum_{i} \epsilon_{i}-\sum_{i, j}\left(2 J_{i j}-K_{i j}\right) .
$$

Moreover, it is quite unrealistic to assume that the true energy $E$, though it is unknown, is expressible in the form of Eq. (9). Thus, the Hartree-Fock wave function never satisfies the zero potential energy criterion [Eq. (2)], which accordingly implies that the Hartree-Fock approximation cannot correctly describe the long-range asymptotic behavior of many-electron wave functions. Though the Hartree-Fock approximation is a well-established independent-particle model and constitutes a beautiful theoretical framework, it may not be very suitable for problems such as scattering and long-range forces if accurate and careful descriptions of the wave function tail are needed. The same is true for the Hartree approximation.

\section{ILLUSTRATIONS}

The majority of atomic and molecular Hartree-Fock calculations uses some basis functions and the (spatial) orbital is approximated by a linear combination of basis functions. Because of the required asymptotic form [Eq. (3)], basis functions other than the Slater and hydrogenic types have no chance to satisfy the zero potential energy criterion. For example, a Gaussian-type function $\chi(\mathbf{r})=f(x, y, z) \exp \left(-\alpha r^{2}\right)$ gives $\epsilon_{\mathrm{zp}}=-\infty$. For this reason, we here examine approximate Hartree-Fock wave functions obtained with Slater-type basis functions.

Table I summarizes the analysis of two types of ground state wave functions for $\mathrm{He}, \mathrm{Be}$, and $\mathrm{Ne}$ atoms. One is the unconstrained wave function due to Clementi and Roetti ${ }^{20}$ and the other is the constrained one due to Weber et al. ${ }^{21}$ and

\begin{tabular}{|c|c|c|c|c|c|c|c|c|}
\hline \multirow[b]{2}{*}{ Atom } & \multicolumn{3}{|c|}{ Orbitalwise } & \multicolumn{2}{|c|}{ Total } & \multicolumn{3}{|c|}{ Zero potential energy criterion } \\
\hline & Orbital & $-\epsilon_{i}$ & $-\epsilon_{\mathrm{p}, i}$ & $-E_{\mathrm{av}}$ & $-E_{\text {rp }}$ & $\begin{array}{l}\text { Orbitalwise } \\
\text { Eq. (4) }\end{array}$ & $\begin{array}{l}\text { Hartree-Fock } \\
\text { Eq. (9) }\end{array}$ & $\begin{array}{l}\text { Exact } \\
\text { Eq. (2) }\end{array}$ \\
\hline \multicolumn{9}{|c|}{ Unconstrained wave functions: } \\
\hline $\mathrm{He}$ & $1 s$ & 0.91795 & 1.00414 & 2.86168 & 2.00829 & No & No & No \\
\hline $\mathrm{Be}$ & $1 s$ & 4.73267 & 0.30280 & 14.57302 & 1.21119 & No & No & No \\
\hline \multirow[t]{3}{*}{$\mathrm{Ne}$} & $1 s$ & 32.77248 & 1.92441 & 128.54705 & 14.02324 & No & No & No \\
\hline & $2 s$ & 1.93043 & 1.92441 & & & No & & \\
\hline & $2 p$ & 0.85044 & 1.05427 & & & & & \\
\hline \multicolumn{9}{|c|}{ Constrained wave functions ${ }^{b}$} \\
\hline He & $1 s$ & 0.91796 & 0.91796 & 2.86168 & 1.83592 & Yes & Yes & No \\
\hline \multirow{2}{*}{$\mathrm{Be}$} & $1 s$ & 4.73279 & 4.73280 & 14.57301 & 10.08416 & Yes & Yes & No \\
\hline & $2 s$ & 0.30929 & 0.30928 & & & Yes & & \\
\hline \multirow[t]{2}{*}{$\mathrm{Ne}$} & $1 s$ & 32.77334 & 32.77334 & 128.54619 & 74.51368 & No & No & No \\
\hline & $2 p$ & 0.85098 & 0.85098 & & & Yes & & \\
\hline
\end{tabular}
Marron et al., ${ }^{22}$ in which the orbitals are forced to satisfy $\zeta_{i}=\left(-2 \epsilon_{i}\right)^{1 / 2}$ asymptotically. The latter authors considered $\zeta_{i}=\left(-2 \epsilon_{h}\right)^{1 / 2}$ to be pathologic while $\zeta_{i}=\left(-2 \epsilon_{i}\right)^{1 / 2}$ to be physically meaningful.

TABLE I. Zero potential energy criteria applied to the Hartree-Fock wave functions for the ground state of He, Be, and Ne atoms.

\footnotetext{
Reference 20 .

${ }^{\mathrm{b}}$ References 21 and 22 .
} 
The unconstrained wave functions reveal one general feature of basis-set-expansion wave functions: In the longrange asymptotic region, the basis function with the smallest exponent governs the behavior of an orbital unless its coefficient of the linear combination is exactly zero. Therefore, the different orbitals $\left\{\psi_{i}\right\}$ expressed by the same basis set have the identical $\epsilon_{\text {zp }},{ }_{i}$. The situation is similar to the orbitalwise criterion [Eq. (4)], but orbitals with different symmetries (e.g., $s$ - and $p$-type orbitals in $\mathrm{Ne}$ atom) can have different $\epsilon_{\mathrm{zp}, i}$ 's, since the exponents are not generally common to basis functions for different symmetries. However, the correct asymptotic behavior [Eq. (3)] suggests that the smallest exponent should be common to all basis sets for different symmetries and be equal to $\left(-2 \epsilon_{h}\right)^{1 / 2}$, where $\epsilon_{h}$ is the energy of the highest occupied orbital. As a result, none of the unconstrained wave function in Table I fulfills the orbitalwise and Hartree-Fock zero potential energy criteria, though the $2 s$ orbitals for $\mathrm{Be}$ and $\mathrm{Ne}$ show relatively good agreement of $\epsilon_{i}$ and $\epsilon_{\mathrm{zp}, i}$.

On the other hand, the constrained wave functions for $\mathrm{He}$ and $\mathrm{Be}$ atoms satisfy both the orbitalwise and HartreeFock criteria. For $\mathrm{Ne}$ atom, however, only the $2 p$ orbital satisfies the orbitalwise criterion, since $\zeta_{i}=\left(-2 \epsilon_{i}\right)^{1 / 2}$ is used as the constraint.

We have also examined several Hartree-Fock wave functions for diatomic molecules calculated by Cade, Huo, and Wahl. ${ }^{23-25}$ The results are similar to those of the unconstrained atomic wave functions given in Table I and no detailed numerical data are presented here.

\section{SUMMARY}

We have shown that the exact zero potential energy criterion [Eq. (2)] cannot be satisfied by the Hartree-Fock wave function. This suggests the Hartree-Fock method may not be suitable for problems in which a very accurate description of the long-range asymptotic behavior is needed. The Hartree-Fock [Eq. (9)] and orbitalwise [Eq. (4)] criteria have been derived, which measure the consistency of the energy and the wave function tail at the level of the Hartree-Fock approximation. The present results are extended straightforwardly to the unrestricted Hartree-Fock wave function: The orbitalwise criterion applies separately to the $\alpha$ and $\beta$ spin orbitals and the Hartree-Fock criterion is expressed as the sum of two contributions, since each of the two Fock equations for the $\alpha$ and $\beta$ spin orbitals has the same asymptotic structure with that for closed shells discussed here.

\section{ACKNOWLEDGMENT}

The author thanks Dr. K. Ohta for helpful discussion.

'T. Koga, J. Chem. Phys. 83, 6301 (1985).

${ }^{2}$ T. Koga and H. Sasaki, Theor. Chim. Acta 72, 27 (1987).

${ }^{3}$ T. Koga, T. Uchiyama, and M. Uji-ie, J. Chem. Phys. 87, 4025 (1987).

${ }^{4}$ J. H. Bartlett, Phys. Rev. 51, 661 (1937).

${ }^{5}$ A. A. Frost, J. Chem. Phys. 10, 240 (1942).

${ }^{6}$ A. A. Frost, R. E. Kellog, and E. C. Curtis, Rev. Mod. Phys. 32, 313 (1960).

${ }^{7}$ B. H. Armstrong, Bull. Am. Phys. Soc. 9, 401 (1964).

${ }^{8}$ R. J. Drachman, Phys. Rev. 136, A641 (1964).

${ }^{9}$ A. J. Thakkar and V. H. Smith, Jr., Phys. Rev. A 18, 841 (1978)

${ }^{10}$ T. Koga, K. Ohta, and A. J. Thakkar, Phys. Rev. A 37, 1411 (1988)

'N. C. Handy, M. T. Marron, and H. J. Silverstone, Phys. Rev. 180, 45 (1969).

${ }^{12}$ G. S. Handler, D. W. Smith, and H. J. Silverstone, J. Chem. Phys. 73, 3936 (1980).

${ }^{13}$ A. J. O’Connor, Commun. Math. Phys. 32, 319 (1973).

${ }^{14}$ J. M. Combes and L. Thomas, Commun. Math. Phys. 34, 251 (1973).

${ }^{15}$ E. H. Lieb and B. Simon, Commun. Math. Phys. 53, 185 (1977).

${ }^{16} \mathrm{M}$. Hoffmann-Ostenhof and T. Hoffmann-Ostenhof, Phys. Rev. A 16, 1782 (1977).

${ }^{17}$ R. Ahlrichs, M. Hoffmann-Ostenhof, and T. Hoffmann-Ostenhof, J. Chem. Phys. 68, 1402 (1978).

${ }^{18}$ T. Hoffmann-Ostenhof, M. Hoffmann-Ostenhof, and R. Ahlrichs, Phys. Rev. A 18, 328 (1978).

19S. H. Patil, J. Chem. Phys. 80, 2689 (1984).

${ }^{20}$ E. Clementi and C. Roetti, At. Data Nucl. Data Tables 14, 177 (1974).

${ }^{21}$ T. A. Weber, N. C. Handy, and R. G. Parr, J. Chem. Phys. 52, 1501 (1970).

${ }^{22}$ M. T. Marron, N. C. Handy, R. G. Parr, and H. J. Silverstone, Int. J. Quantum Chem. 4, 245 (1970).

${ }^{23}$ P. E. Cade and W. Huo, At. Data Nucl. Data Tables 12, 415 (1973).

${ }^{24}$ P. E. Cade and A. C. Wahl, At. Data Nucl. Data Tables 13, 339 (1974).

${ }^{25}$ P. E. Cade and W. M. Huo, At. Data Nucl. Data Tables 15, 1 (1975). 